\title{
Research on the Set Curve for Tandem Cold Rolling Mill Automatic Flatness Control
}

\author{
Xiaomin Zhou ${ }^{1, \text { a }}$, Xiaoxue Yue ${ }^{1, b}$ \\ ${ }^{1}$ School of Mechanical Engineering, University of Science and Technology Beijing, Beijing 100083 \\ aemail: zhouxiaomin@ustb.edu.cn, bemail:542757719@qq.com
} Keywords: Cold Rolling Mill, Automatic Flatness Control, Set Curve, Uneven Temperature
Distribution

\begin{abstract}
Flatness set curve is the target of strip flatness control system which represents the desired strip flatness quality. If the longitudinal residual stress along the transverse distribution of the strip can be measured accurately by the flatness measuring roll, the actual flatness can be controlled accurately. However the actual strip flatness is different from the online measured flatness because of the various influence factors. The purpose of the flatness set curve is to compensate these factors and to satisfy the requirements of next lines. The optimizations of the set curve are proposed for the uneven temperature distribution of the rolled strip in this paper. Verified by the rolling experiments, the strip flatness quality is improved.
\end{abstract}

\section{Introduction}

In strip cold rolling process, the longitudinal extension in the width direction of strip is often uneven because of the various influence factors. So the uneven longitudinal residual stress along the transverse distribution is produced $[1,2]$. It may cause the strip flatness defects if the uneven distribution of residual stress differences exceed a certain range [3].

Flatness set curve is the target of strip flatness control system which represents the desired strip flatness quality by the producers or the next lines $[4,5]$. If the longitudinal residual stress along the transverse distribution of the strip can be measured correctly by the flatness measuring roll corresponding to the actual tensile stress transverse distribution, the actual flatness can be controlled accurately. However, because of the various influence factors, the actual strip flatness after rolling is different from the online measured flatness [6]. The purpose of the flatness set curve is to compensate these factors and to satisfy the special requirements of the subsequent lines.

The research on flatness set curve has been in progress since the close loop control of flatness was proposed in cold rolling mills [7,8]. The development of the flatness set curve has involved several stages including the target of no buckling wave, the non-flatness signals compensation of the measuring flatness, the requirements consideration of the next lines etc. $[9,10]$.

The optimizations of the set curve are proposed for the uneven temperature distribution by analyzing the influence factors of the set curve and tracking the temperature of the rolled strips in this paper. The optimization performances are verified by the rolling experiments. The strip flatness quality is improved.

\section{The analysis of the flatness set curve}

There are five stands in the experimental tandem cold rolling mills. All of five stands are equipped with CVC6 settings. The flatness control actuators includes the positive and negative bending of the work roll and intermediate roll, shift of the intermediate roll and 25 district zones cooling of the work roll. The flatness measurement instrument is BFI tensile stress measurement roll provided by Siemens, installed after the fifth stand to implement the close loop control system.

There are two ways to establish the set curve of the automatic flatness control system. One is to create 80 desired flatness values along the strip width. The set values of the flatness can be adjusted point by point. So any form of the set curve can be achieved by this method. The second method is 
to create the set curve by three parameters including the constant term, the quadratic term and quartic term coefficients of the standard polynomial curve expressions.

The flatness set curve is also considered for the different requirements according to the subsequent process. The set curve is considered to be micro edge wave form for the next batch annealing lines in order to keep the evenly heating and hold the uniform interlayer pressure in the coil batch annealing process. And the edge wave can be minimized by the next temper mills. The set curve is considered to be micro center wave form for the next continuous annealing lines in order to keep the even tension distribution along the width direction. The set curve of the final product can be considered as flat or micro center wave. The set curve is also smaller than the buckling instability threshold of the strip [11].

In the actual production process, flatness set curve can be modified online through the "Tilt", "Bend", "Ed" buttons on HMI interface. The modification is mainly to compensate for the various influences including the uneven temperature distribution, the deflection and geometric misalignment of the flatness measurement roll, the change of the coil outer profile etc. But this modification rely on the experiences of the technicians.

Through online measurement and statistical analysis, the set curve correction by the "Tilt" is shown as the equation (1) as follows.

$$
f_{1}(x)=20 \cdot " \text { Tilt". } x
$$

Through online measurement and statistical analysis, the set curve correction by the "Bend" is shown as the equation (2) as follows.

$$
f_{2}(x)=80 \cdot \text { " Bend ". }\left(x^{2}-0.125\right)
$$

Through online measurement and statistical analysis, the set curve correction by the "Ed" is shown as the equation (3) as follows.

$$
f_{\text {ed }}(x)=" \operatorname{Ed}^{\prime \prime} *(\text { e1, e2, e3, e4, e5) }
$$

Where: (e1,e2, e3, e4, e5) are the edge compensation factors, and the values are $(20,12,6,2,0) \circ$

The set curve $f(x)$ actually applied in the automatic flatness control system is shown as the equation (4) as follows.

$$
f(x)=f_{0}(x)+f_{1}(x)+f_{2}(x)+f_{\text {ed }}(x)
$$

Where $f_{0}(x)$ is the set curve created by the 80 desired either flatness values or three parameters.

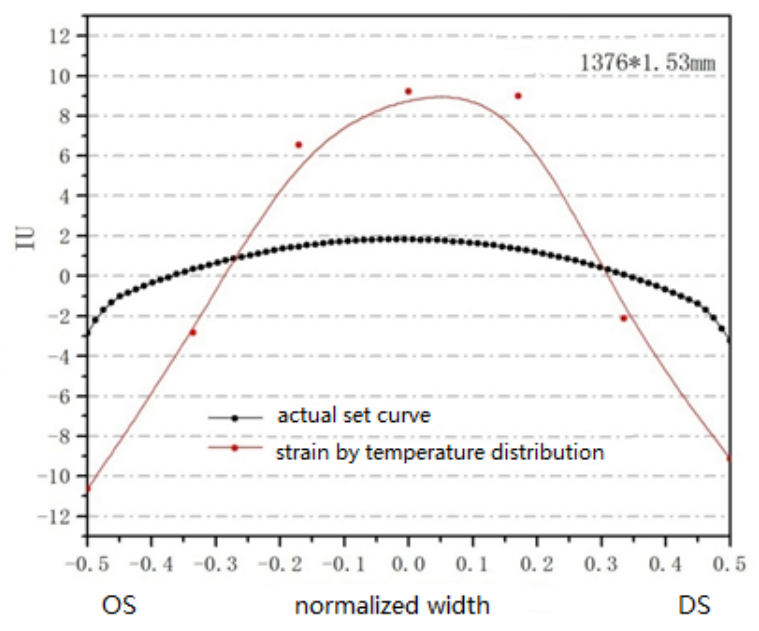

Fig.1 The applied set curve in the process

The actually applied set curve in the process and the strain caused by the actual temperature distribution of the rolled strip are shown in the Fig.1. It shows that the applied set curve cannot compensate the uneven temperature distribution adequately. It means that the compensation is not 
enough for the actual temperature uneven distribution. So the edge wave is produced easily.

\section{The optimization of the flatness set curve}

There is the temperature difference in the strip width direction due to the deformation in the rolling process. It will cause the uneven thermal expansion along the width direction and reflect the transversely uneven temperature additional stress of the winding tension. This uneven temperature additional stress is proportional to corresponding temperature difference. The transversely temperature difference of $1{ }^{\circ} \mathrm{C}$ will lead to $2.5 \mathrm{Mpa}$ stress difference (or 1.2IU) under certain conditions.

By analyzing the large number of measured strip temperature data, the temperature compensation is statistically determined based on the assumption of the relatively stable rolling process. The parts of the strip temperature data are shown in Fig.2.

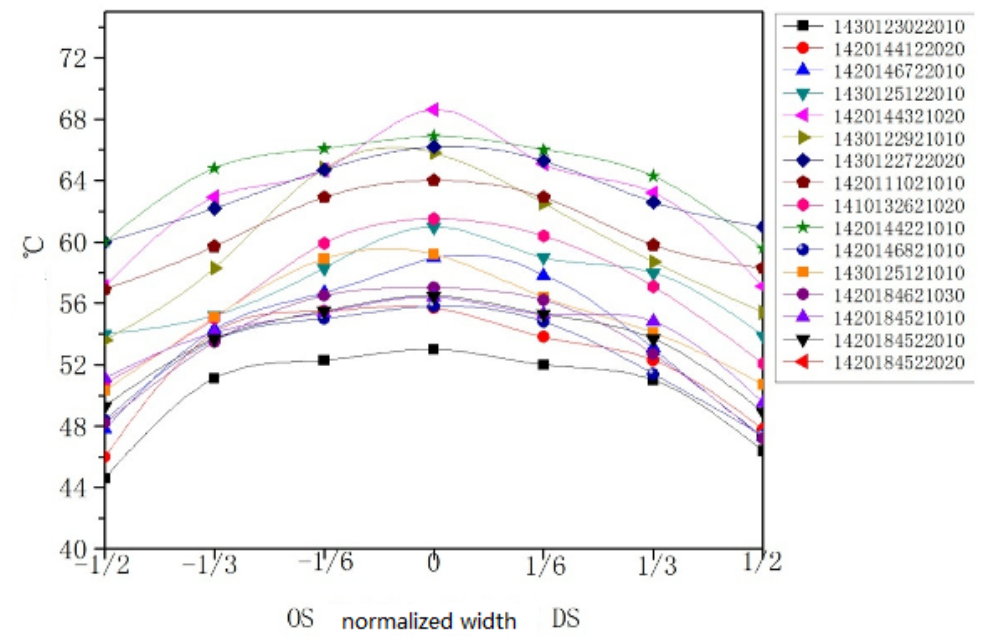

Fig.2 The actual strip temperature distribution

The least squares method is used for the measured discrete temperature data. The temperature distribution function along the width direction can be obtained as equation (5) as follows based on the incomplete quartic polynomial fitting.

$$
\mathrm{T}(x)=A_{0}+A_{1} x+A_{2} x^{2}+A_{4} x^{4}
$$

Where A0, A1, A2, A4 are fitting coefficients. $\mathrm{x}$ is normalized horizontal coordinate.

The transversely additional thermal $\operatorname{strain} \varepsilon(\mathrm{x})$ can be calculated as equation (6) as follows by the strip temperature distribution function $\mathrm{T}(x)$.

$$
\varepsilon(\mathrm{x})=-\alpha \cdot \mathrm{T}(x)
$$

Where the linear expansion coefficient $\alpha=1.2 \times 10^{-5} /{ }^{\circ} \mathrm{C}$.

According the actual temperature distribution difference, the fitting temperature distribution function $T(x)$ is shown as equation (7) as follows.

$$
\mathrm{T}(x)=3.40+0.52 x-38.21 x^{2}+33.32 x^{4}
$$

And the corresponding additional thermal strain $\varepsilon(x)$ can be obtained as equation (8) as follows.

$$
\varepsilon(x)=3.97+0.60 x-44.71 x^{2}+38.98 x^{4}
$$




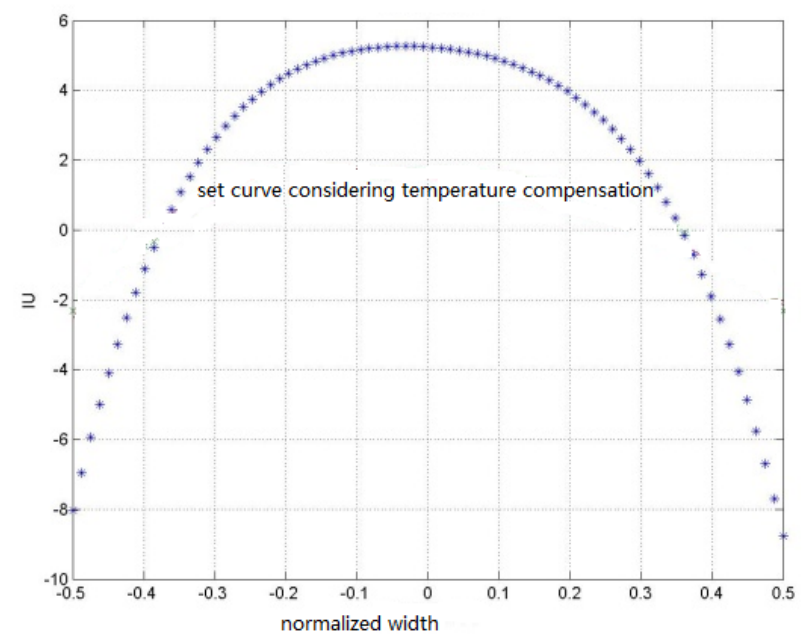

Fig. 3 The set curve considering the temperature compensation

The flatness set curve considering the compensation of the uneven temperature distribution is shown as Fig.3 as follows. It is smaller than the buckling instability threshold of the strip by simulation verification.

From the experiment results in the experimental 1970CVC cold rolling mills, the percent of flatness smaller than 3IU is improved by using new set curve. From the response of the next continuous annealing lines, the edge wave situation is improved by using the new set curve. The flatness quality of the strip is enhanced.

\section{Conclusion}

For the flatness issues caused by uneven temperature distribution, the set curve compensation is analyzed and achieved according to the actual strip temperature by measurement and statistical analysis. From the experiment results in the cold rolling mills, the percent of flatness smaller than 3IU is improved by using new set curve. From the response of the next continuous annealing lines, the edge wave situation is improved by using the new set curve. The flatness quality of the strip is improved.

\section{Acknowledgement}

In this paper, the research was sponsored by the National Natural Science Foundation of China (Project No. 51204017).

\section{References}

[1] ZHANG Qing-dong. WU Yue.et al. Improving on the Flatness Control Performance of 1220mm Cold Tandem Mill[J].Shanghai Metals.2005.27(3):23 25.

[2] DAI Jiang-bo. WU Wen-bin, ZHANG Qing-dong.et al. Recognition and Evaluation System for Strip Flatness on 2030mm Cold Tandem Mills[J].Journal of University of Science and Technology Beijing.2003.25(6):572 575.

[3] XU Jian-yong .JIANG Zheng-lian.QUE Yue-hai. Effect of Incoming Hot Rolled Slab and Cold Rolling Technology on Mill Exit Strip Shape [J].China Metallurgy.2003.15(5):60 64.

[4] JIA Sheng-hui. ZHANG Jie. CAO Jian-guo.et al. Flatness Object Curve for Non-tropism Silicon Steel Rolling in 5-stand Tandem Cold Rolling Mill. Journal of University of Science and Technology Beijing.2002.24(3):303 305.

[5] JIA Chun-yu.SHANG Zhi-dong. Compensation of Plank Shape Target Curves for Cold-Rolling Strip [J].Journal of Iron and Steel Research. 2000.12(4):64 67. 
[6]YANG Quan. Study on the Rolled Buckling and the Target Shape in the Automatic Flatness Control [D].Beijing: University of Science and Technology Beijing. 1992:43 68 .

[7]Wang Dongcheng, Liu Hongmin. Flatness On-Line Preset Technology for Cold Rolling Mill[C]. Advanced Materials Research .2012, 572:120-124.

[8]Wang Dongcheng. A Model Coupling Method for Shape Prediction [J]. Journal of Iron and Steel Research, International, 2012,19(6):19-24.

[9]Neto L S, Ayhan T. Coil Build Up Compensation During Cold Rolling to Improve Off-line Flatness [J]. Light Metals, 2011, 621-624.

[10]Sun Yabo, Liu Hongmin, Peng Yan. Establishment Method of Shape Standard Curve Based on Stepwise Optimization and Its Compensation Models [J]. Chinese Journal of Mechanical Engineering, 2011,01:105-112.

[11]Kim J, Pattnaik S. Prediction of Formation of Wavy Surfaces in Rolled Plates by Post-buckling Analysis [J]. J Appl Mech-Trans ASME, 2010,77: 1-8. 